\title{
Heme oxygenase is not involved in the anti-proliferative effects of statins on pancreatic cancer cells
}

\author{
K. Vanova', S. Boukalova², H. Gbelcova ${ }^{3}$, L. Muchova', J. Neuzil ${ }^{2,4}$, R. Gurlich ${ }^{5}$, T. Ruml ${ }^{3}$ and L. Vitek ${ }^{1,6^{*}}$
}

\begin{abstract}
Background: Pancreatic cancer is recognized as one of the most fatal tumors due to its aggressiveness and resistance to therapy. Statins were previously shown to inhibit the proliferation of cancer cells via various signaling pathways. In healthy tissues, statins activate the heme oxygenase pathway, nevertheless the role of heme oxygenase in pancreatic cancer is still controversial. The aim of this study was to evaluate, whether anti-proliferative effects of statins in pancreatic cancer cells are mediated via the heme oxygenase pathway.
\end{abstract}

Methods: In vitro effects of various statins and hemin, a heme oxygenase inducer, on cell proliferation were evaluated in PA-TU-8902, MiaPaCa-2 and BxPC-3 human pancreatic cancer cell lines. The effect of statins on heme oxygenase activity was assessed and heme oxygenase-silenced cells were used for pancreatic cancer cell proliferation studies. Cell death rate and reactive oxygen species production were measured in PA-TU-8902 cells, followed by evaluation of the effect of cerivastatin on GFP-K-Ras trafficking and expression of markers of invasiveness, osteopontin (SPP1) and SOX2.

Results: While simvastatin and cerivastatin displayed major anti-proliferative properties in all cell lines tested, pravastatin did not affect the cell growth at all. Strong anti-proliferative effect was observed also for hemin. Co-treatment of cerivastatin and hemin increased anti-proliferative potential of these agents, via increased production of reactive oxygen species and cell death compared to individual treatment. Heme oxygenase silencing did not prevent pancreatic cancer cells from the tumor-suppressive effect of cerivastatin or hemin. Cerivastatin, but not pravastatin, protected Ras protein from trafficking to the cell membrane and significantly reduced expressions of SPP1 $(p<0.05)$ and SOX2 $(p<0.01)$.

Conclusions: Anti-proliferative effects of statins and hemin on human pancreatic cancer cell lines do not seem to be related to the heme oxygenase pathway. While hemin triggers reactive oxygen species-induced cell death, cerivastatin targets Ras protein trafficking and affects markers of invasiveness.

Keywords: Heme, Heme oxygenase, Pancreatic cancer, Statins

\section{Background}

Pancreatic cancer has a very poor prognosis mainly due to late diagnosis of already advanced tumors, often with metastases to distant organs. Since high resistance to therapy aggravates the treatment outcomes, new efficient treatment modalities and therapy targets are under investigation.

\footnotetext{
* Correspondence: vitek@cesnet.cz

${ }^{1}$ Institute of Medical Biochemistry and Laboratory Diagnostics, 1st Faculty of Medicine, Charles University in Prague, Katerinska 32, Prague 2120 00, Czech Republic

${ }^{6} 4$ th Department of Internal Medicine, 1st Faculty of Medicine, Charles University in Prague, Katerinska 32, Prague 2120 00, Czech Republic
} Full list of author information is available at the end of the article
Statins, competitive inhibitors of 3-hydroxyl-methylglutaryl coenzyme A (HMG CoA) reductase, are widely used for treatment of hypercholesterolemia. However, their therapeutic role surpasses the cholesterol lowering capacity, utilizing anti-inflammatory, anti-oxidant and anti-thrombotic actions [1]. Additionally, several studies suggested the antiproliferative role of statins in various cancer cell lines, including lung [2], colorectal [3] and pancreatic cancer [4-7]. These effects could be partly mediated by the depletion of several important intermediates of cholesterol biosynthesis involved in posttranslational protein prenylation. This process is especially important for modification of small GTPases, such as Ras $[8,9]$, which is essential for their 
translocation from cytoplasm to the cell membrane, affecting thus their cell proliferating activities [1] via targeting several important signal transduction pathways [10-12]. The association of activation mutations in the K-ras oncogene with pancreatic cancer is well established, being found in more than $90 \%$ of human pancreatic cancers [13]. We previously reported that most statins protect green fluorescent protein (GFP)-K-Ras from its anchoring to the cell membrane, affecting the signaling pathways and leading to suppression of cancer cell growth in pancreatic cancer cells in vitro [4].

Heme oxygenase (HMOX), the key enzyme in heme metabolism, catalyzes the degradation of heme to equimolar quantities of $\mathrm{CO}$, free iron and biliverdin, which is subsequently converted to bilirubin [14]. While the induction of HMOX1 represents a key biological process in adaptive response to cellular stress and displays antiinflammatory, anti-apoptotic and anti-oxidative actions [14-17], its role in cell proliferation and tumor progression is still controversial $[18,19]$. Some studies suggested that statins can upregulate the $H M O X$ gene expression in a cell- and species-specific manner [20-24], and they exert some of their protective effects via this pathway [21]. However, the upregulation of HMOX1 in pancreatic cancer cells was previously connected to worsened treatment outcome [25].

The aim of this study was to evaluate anti-proliferative effects of statins with respect to their possible role in modulation of HMOX pathway in pancreatic cancer in vitro. Hemin, a strong HMOX1 inducer [26], was used a control compound. Further, we investigated the effects of cerivastatin on targeting the GFP-K-Ras protein trafficking, as well as the regulation of invasiveness of pancreatic adenocarcinoma cells in vitro, elucidating the potential involvement of statins in pancreatic cancer therapy.

\section{Methods \\ Chemicals}

Cerivastatin, pravastatin and fluvastatin were purchased from LKT Laboratories, Inc (USA), lovastatin and simvastatin from Santa Cruz Biotechnology (Dallas, TX, USA). Bovine serum albumin (BSA), hemin, reduced nicotinamide adenine dinucleotide (NADPH), sulfosalicylic acid, Dulbecco's Modified Essential Media (DMEM), and RPMI-1640 were purchased from Sigma-Aldrich (St. Louis, MO, USA). Fetal bovine serum (FBS) and Lglutamine (L-Glu) were purchased from Biosera (Boussens, France), 15-deoxy- $\Delta$-12,14-prostaglandin J2 (PGJ2) was purchased from Merck (Darmstadt, Germany).

\section{Cell culture}

For cell culture studies, the following pancreatic cancer cell lines were used: PA-TU-8902 (DSMZ, Braunschweig,
Germany), MiaPaCa-2 and BxPC-3 (ATCC, Manassas, VA, USA). All cell lines were maintained and grown in a humidified atmosphere containing $5 \% \mathrm{CO}_{2}$ at $37{ }^{\circ} \mathrm{C}$. PATU-8902 and MiaPaCa-2 were cultured in DMEM supplemented with $10 \%$ FBS, antibiotics and $1 \%$ L-Glu, BxPC-3 in RPMI-1640 supplemented with $10 \%$ FBS, antibiotics and $2 \%$ L-Glu. For all experiments, medium with reduced content of FBS to the final concentration of $0.5 \%$ was used. All statins in the study were used at $12 \mu \mathrm{M}$ (corresponding to $\mathrm{IC}_{50}$ of simvastatin for $\mathrm{MiaPaCa}-2$ cells after $24 \mathrm{~h}$ incubation [4]) diluted in methanol (vehicle) and hemin (methemalbumin) was prepared as previously described and used in the final concentration of $30 \mu \mathrm{M}$ $(\mathrm{pH}=7.4)[26]$.

Ethical approval for work on cell lines was not required by our Institution.

\section{HMOX RNA interference (RNAi)}

Pancreatic cancer cells were transfected with 10 pmol of HMOX1 esiRNA and 10 pmol of HMOX2 esiRNA (Sigma-Aldrich) per $5 \times 10^{3}$ seeded cells using the Lipofectamine RNAiMAX reagent (Life Technologies, Carlsbad, CA, USA) for $24 \mathrm{~h}$ in ATB-free DMEM medium. The esiRNA Universal control was used as negative control in all experiments. Data were expressed as \% of esiRNA Universal control (Sigma-Aldrich).

\section{Cell proliferation assay}

For the cell proliferation assay, cells were seeded into 96 well (5-12.5 x $10^{4}$ cells per $\mathrm{ml}$ according to the cell line) and kept at $37{ }^{\circ} \mathrm{C}$ and $5 \% \mathrm{CO}_{2}$. After $24 \mathrm{~h}$, cells were treated with statins or/and hemin, followed by the MTT test (Sigma-Aldrich) as a general cell proliferation assay. As we experienced difficulties with hemin-treated samples using MTT test due to interfering effects of hemin, we further used the more sensitive CellTiter-Glo Luminescent Cell Viability Assay (Promega, Fichburg, WI, USA). Both tests were used according to the manufacturer's instructions. Results were expressed as \% of controls.

\section{HMOX activity measurement}

Cells in plates were treated with statins and hemin. After $12 \mathrm{~h}$, cells were washed twice with ice-cold phosphate buffer and finally collected into freshly added phosphate buffer and centrifuged. The pellet was resuspended in $150 \mu \mathrm{l}$ of $0.1 \mathrm{M}$ potassium phosphate buffer $(\mathrm{pH}=7.4$ ) and sonicated with an ultrasonic cell disruptor (Model XL2000, Misonics, Farmingdale, NY, USA). The protein concentration was assessed using the $\mathrm{DC}^{\mathrm{T}}$ Protein Assay (Bio-Rad Laboratories, Hercules, CA, USA) according to the manufacturer's instruction. A total of $0.15 \mathrm{mg}$ of protein was incubated for $15 \mathrm{~min}$ at $37{ }^{\circ} \mathrm{C}$ in $\mathrm{CO}$-free septum-sealed vials containing $20 \mu \mathrm{l}$ of $4.5 \mathrm{mM}$ NADPH 
as previously described [27]. The amount of $\mathrm{CO}$ generated by HMOX activity was quantified by gas chromatography with a reduction gas analyzer (Peak Laboratories LLC, Mountain View, CA, USA) and calculated as pmol $\mathrm{CO} / \mathrm{h} / \mathrm{mg}$ protein. Five $\mu \mathrm{M}$ PGJ2 was used as a positive control of heme regulation. Results were expressed as \% of control.

\section{Western blot analyses}

For protein expression analyses, cells were transfected with esiRNA universal control or esiRNA HMOX1/2 as mentioned previously. After $24 \mathrm{~h}$, cells were treated with $30 \mu \mathrm{m}$ hemin for $20 \mathrm{~h}$. Hemin treatment was used to upregulate HMOX1 protein expression to cumulate detectable levels of HMOX1 protein. Thirty $\mu \mathrm{g}$ of total protein were separated on $12 \%$ polyacrylamide gel and then transferred to nitrocellulose membrane (Bio-Rad Laboratories). After blocking in Tween-PBS with $5 \%$ milk (Sigma-Aldrich) for at least $1 \mathrm{~h}$, membranes were incubated with HMOX1 antibody (1:1000; Thermo Fisher, Rockford, IL, USA), or $\beta$-actin (1:1000; Cell Signaling Technology, Danvers, MA, USA) overnight at $4{ }^{\circ} \mathrm{C}$. After washing, membranes were incubated with anti-mouse IgG-HRP (Abcam, Cambridge, UK) for $1 \mathrm{~h}$. Immunocomplexes on the membranes were visualized with ECL Western Blotting Detection Reagents (Cell Signaling Technology).

\section{Real-time PCR analysis of mRNA HMOX1 expression}

Cells grown in plates were treated with statins, hemin or PGJ2. After $4 \mathrm{~h}$, they were washed twice with ice-cold PBS and collected in the lysis buffer. Total cell RNA was isolated using Perfect Pure RNA Cultured Cell Kit (5Prime, Gaithersburg, MD, USA) and cDNA was generated using High Capacity RNA-to-cDNA Master Mix (Life Technologies) according to the manufacturer's instructions. Real-time PCR for HMOX1 (OMIM *141250) and HMOX2 (OMIM *141251) was performed using the SYBR master mix (Life Technologies) according to the manufacturer's instructions with optimized primers (Generi Biotech, Hradec Králové, Czech Republic). Results were calculated using the comparative Ct method with $H P R T$ as a house-keeping gene and were expressed as \% of control.

\section{Markers of invasiveness}

Cells were treated for 12, 24 and $48 \mathrm{~h}$ with individual statins. Total RNA was collected and cDNA generated as mentioned above. For real-time qPCR, cDNA corresponding to $10 \mathrm{ng}$ of starting total RNA was diluted with water in $3.6 \mu \mathrm{l} ; 0.2 \mu \mathrm{l}$ of the combined $10 \mu \mathrm{M}$ forward and reverse primers were added and, finally, $3.8 \mu \mathrm{l}$ of $2 \mathrm{x}$ iTaq Universal SYBR Green Supermix (Bio-Rad Laboratories) was added. The reaction was carried out using the Eco real-time PCR system (Illumina, San Diego, CA, USA) using three-step PCR. The relative mRNA expression levels of osteopontin (secreted phosphoprotein 1, SPP1, OMIM*166490) and sex-determining region Y-related HMG box 2 (SOX2, OMIM*184429) were calculated using the comparative $\mathrm{Ct}(\Delta \Delta \mathrm{Ct})$ method, with ribosomal phosphoprotein (P0, OMIM*180510) as a reference gene.

Sequences of primers used for real-time PCR: SPP1 forward, AGA CCT GAC ATC CAG TAC CCT, reverse - CAA CGG GGA TGG CCT TGT AT; SOX2 forward AGG ACC AGC TGG GCT ACC CG, reverse - GCC AAG AGC CAT GCC AGG GG.

\section{Apoptosis evaluation}

Apoptosis was quantified using the annexin V-FITC method, which detects phosphatidyl serine externalized in the early phases of apoptosis, in combination with propidium iodide (PI) staining. After exposure to cerivastatin and/or hemin, floating and attached cells were collected, washed with PBS, re-suspended in $100 \mu \mathrm{l}$ binding buffer and incubated for $20 \mathrm{~min}$ at room temperature with $0.3 \mu \mathrm{l}$ annexin V-FITC (Apronex, Vestec, Czech Republic). PI (10 $\mu \mathrm{g} / \mathrm{ml})$ was added directly before flow cytometry analysis (BD FASC Calibur, BD, Franklin Lakes, NJ, USA). Annexin V positive $(\mathrm{An}+)$ and PI negative (PI-) are cells in early apoptosis, An + and/or PI positive (PI+) are cells in late apoptosis or post-apoptotic necrosis.

\section{Reactive oxygen species (ROS) generation}

For assessment of ROS generation, dichlordihydrofluorescein diacetate (H2DCFDA) (Life Technologies) was used. After treatment, cells were washed and exposed to $10 \mu \mathrm{M}$ H2DCFDA in $37^{\circ} \mathrm{C}$ for $20 \mathrm{~min}$. Cells were then washed, lysed and the fluorescent signal at 492/520 (Ex/ $\mathrm{Em})$ was evaluated in $100 \mu \mathrm{l}$ aliquots. Total fluorescence was related to protein concentration. Results were expressed as \% of control.

\section{Ras protein translocation assay}

PA-TU-8902 cells were seeded in dishes with glass bottom $6 \mathrm{~h}$ before transfection by pEGFP-KrasWT (GFP - green fluorescent protein, WT-wild type) plasmid prepared as described previously [4]. Transfection was carried out using FuGene HD according to the manufacturer's instructions. Cerivastatin $(12 \mu \mathrm{M})$, pravastatin $(12 \mu \mathrm{M})$ and hemin $(30 \mu \mathrm{M})$ were added $12 \mathrm{~h}$ post transfection and the cells incubated with the agents for $24 \mathrm{~h}$. Intracellular localization of the GFP-K-Ras protein was visualized by confocal microscopy, using a spinning disk confocal microscope (Olympus, Tokyo, Japan; Andor, Belfast, UK) equipped with solid state laser (488 $\mathrm{nm}$ for continual excitation). Emission was collected through a single-band 
filter (BrightLine ${ }^{\oplus}$ FF01-525 nm, Semrock Inc., NY, USA). The images were obtained and analyzed with the iQ2 software (Andor).

\section{Statistical analysis}

All data were expressed as mean \pm SD. For normally distributed datasets, one-way ANOVA with post-hoc Holm-Sidak test for multiple comparisons was used for analysis. For non-normally distributed data and small datasets $(n \leq 6)$, Mann-Whitney rank sum test and Kruskal-Wallis ANOVA with Dunn's test for multiple comparisons were used. $P$-values less than 0.05 were considered statistically significant.

All datasets of the results discussed in the manuscript are available on request.

\section{Results}

Statins exert different anti-proliferative on pancreatic cancer cell lines

The inhibitory effects of individual statins on proliferation were assessed using cultured human pancreatic cancer cells. Except pravastatin, all selected statins, used at $12 \mu \mathrm{M}$, showed significant anti-proliferative effects on growth of tested cancer cells after $48 \mathrm{~h}$ of treatment (Fig. 1). Various statins exhibited different antiproliferative potential covering the spectrum from the most effective cerivastatin and simvastatin, followed by fluvastatin and lovastatin, to ineffective pravastatin. Additionally, we observed significantly different sensitivity for particular pancreatic cancer cell lines. The most sensitive cell line was $\mathrm{MiaPaCa}-2$, bearing activating $\mathrm{K}$-ras mutation in codon 12 (34G > T) [28]. By contrast, the other cell line PA-TU-8902 with another activating mutations in K-ras oncogene in codon 12 (35G > T) [29] revealed to be more resistant to statin treatment. BxPC-3 cells, featuring wild-type $K$-ras oncogene and overexpressing cyclo-oxygenase-2 [28], were slightly more sensitive than PA-TU-8902 cells to several statins and significantly more resistant to statin treatment than MiaPaCa-2 cells (Fig. 1). The most efficient statin was cerivastatin, which decreased cell proliferation as compared to untreated controls to $51 \pm 8 \%, 61 \pm 10 \%$, and $14 \pm 2 \%$ following 48-h treatment of PA-TU-8902, BxPC-3 and MiaPaCa-2 cells, respectively, with $p<$ 0.0001 for all comparisons.

\section{Statins do not affect HMOX expression and activity in human pancreatic cancer cells}

To find out whether statins regulate HMOX activity in selected pancreatic cell lines in vitro, and whether this mechanism could possibly contribute to their antiproliferative properties, we treated pancreatic cancer cells with individual statins for $12 \mathrm{~h}$. However, none of the statins affected HMOX activity in any of the studied

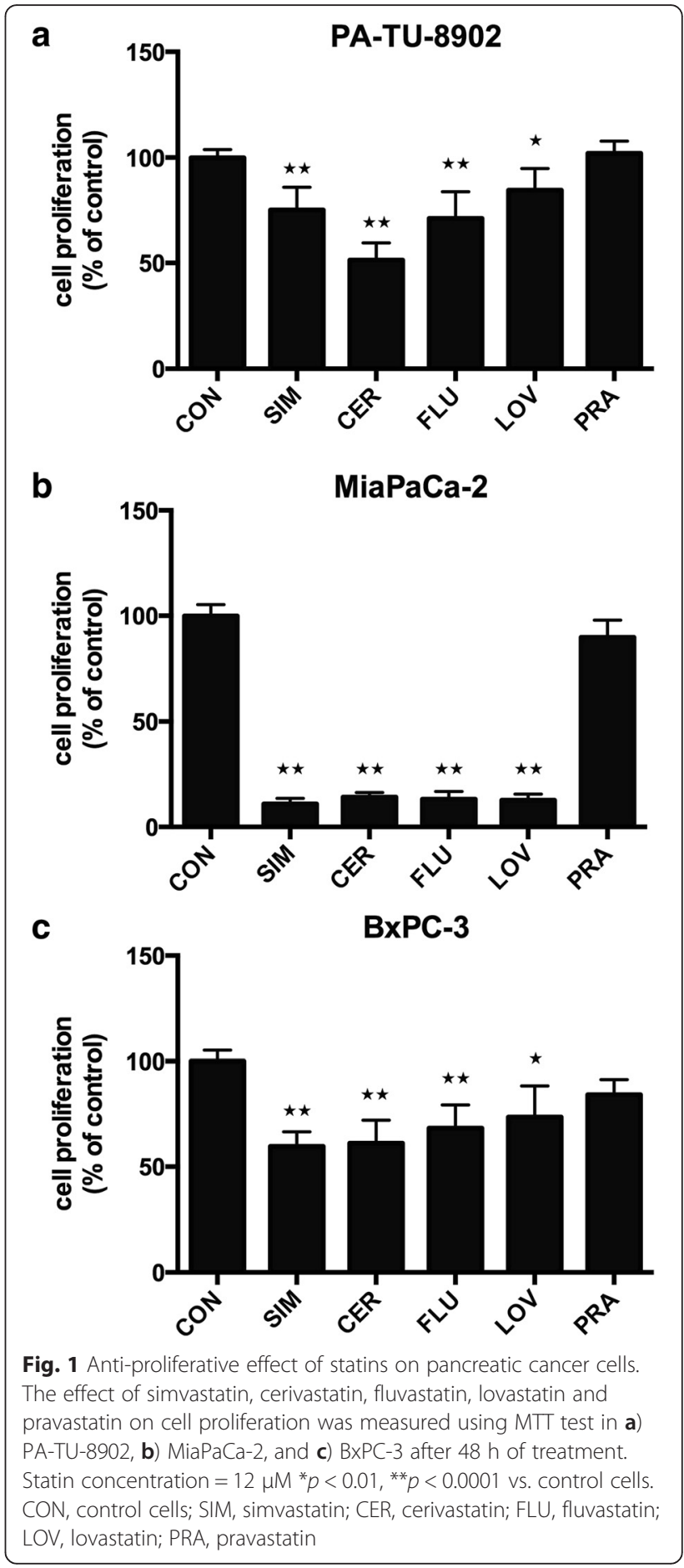

cell lines (Fig. 2a). There was no difference in basal HMOX activities between PA-TU-8902, MiaPaCa-2 and BxPC-3 cells $(0.79 \pm 0.12$ vs. $0.89 \pm 0.19$ vs. $0.90 \pm$ $0.14 \mathrm{nmol} \mathrm{CO} / \mathrm{h} / \mathrm{mg}$ protein, respectively, $p>0.05)$. For detailed analysis, we selected the most resistant cell line, PA-TU-8902, and tested the effect of cerivastatin, the most efficient statin (Fig. 1). Hemin and PGJ2, used as positive controls, strongly increased both expression and 


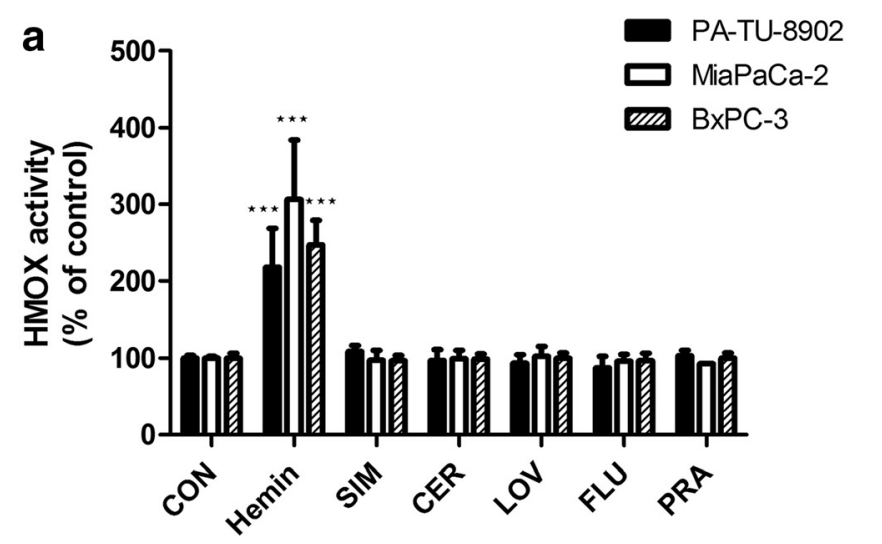

b

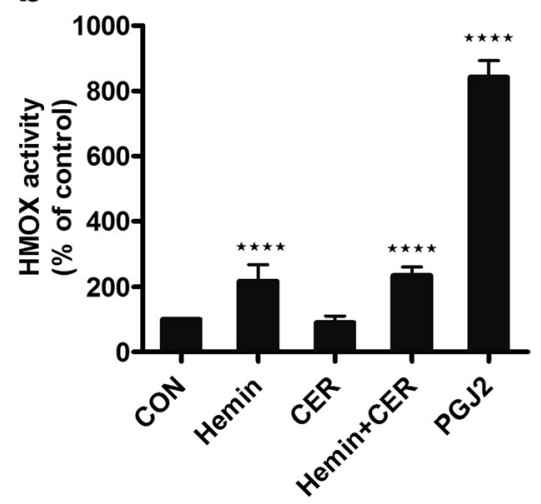

c
PA-TU-8902

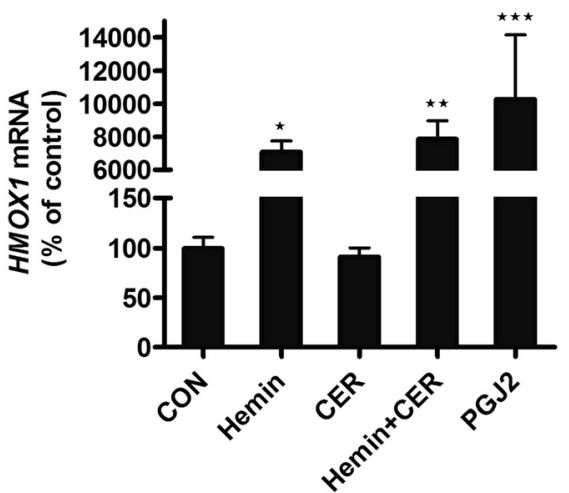

Fig. 2 The effect of statins on activity and expression of HMOX in pancreatic cancer cells a) HMOX activity was measured in PA-TU-8902, MiaPaCa-2 and BxPC-3 pancreatic cancer cell lines after $12 \mathrm{~h}$ of statin treatment $(12 \mu \mathrm{M})$. $\mathbf{b}$ HMOX activity after $12 \mathrm{~h}$ of treatment were measured in

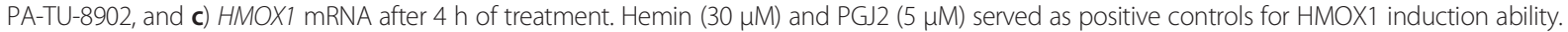
${ }^{*} p<0.05,{ }^{* *} p<0.01,{ }^{* * *} p<0.001,{ }^{* * * *} p<0.0001$ vs. control cells CON, control cells; SIM, simvastatin; CER, cerivastatin; FLU, fluvastatin; LOV, lovastatin; PRA, pravastatin; PGJ2, 15-deoxy- $\Delta$-12,14-prostaglandin $J 2$

activity of HMOX after 4 and $12 \mathrm{~h}$, respectively. Cerivastatin affected neither HMOX1 expression nor HMOX activity in PA-TU-8902 cells. Addition of cerivastatin to hemin had no impact on HMOX induction by hemin itself (Fig. 2b, c).

Hemin augments anti-proliferative effects of selected statins in pancreatic cancer cells

While using hemin primarily as a HMOX inducer, we unexpectedly noticed the decrease in proliferation in hemin-treated cells. Thus we further focused on more detailed analysis of possible anti-proliferative effects of hemin on the growth of pancreatic cancer cell lines. Indeed, we found a significant effect of $30 \mu \mathrm{M}$ hemin on cell proliferation in all used cell lines after $48 \mathrm{~h}$ (Fig. 3), which was dose-dependent (data not shown). Hemin treatment decreased cell proliferation to $62 \pm 5 \%, 51 \pm 3 \%$, and $38 \pm 8 \%$ in PA-TU-8902, BxPC-3 and MiaPaCa-2 cancer cells, respectively, with $p<0.0001$ for all comparisons. Furthermore, we observed enhancement of anti- proliferative effects of statins by hemin, documented as decreased cell proliferation after $48 \mathrm{~h}$ of cotreatment (Fig. 3).

\section{Suppression of HMOX gene expression does not diminish} anti-proliferative effects of cerivastatin and hemin

Recently it has been suggested, that statin treatment promotes post-transcriptional regulation of the HMOX1 protein rather than affecting $H M O X 1$ gene expression in human endothelial cells [30]. To further resolve if HMOX is involved in anti-proliferative effects of statins on human pancreatic cancer, we silenced $H M O X 1$ and HMOX2 in pancreatic cancer cells with esiRNA and assessed cell proliferation after $48 \mathrm{~h}$ of exposure to cerivastatin and hemin. In all cancer cell lines, $H M O X 1$ and $H M O X 2$ were successfully silenced as demonstrated in Fig. 4. Significant anti-proliferative effect of cerivastatin was observed after $48 \mathrm{~h}$ in both silenced and cells transfected with esiRNA universal control. Interestingly, cell growth was affected by the silencing itself implying the important role of HMOX in the cell survival. 


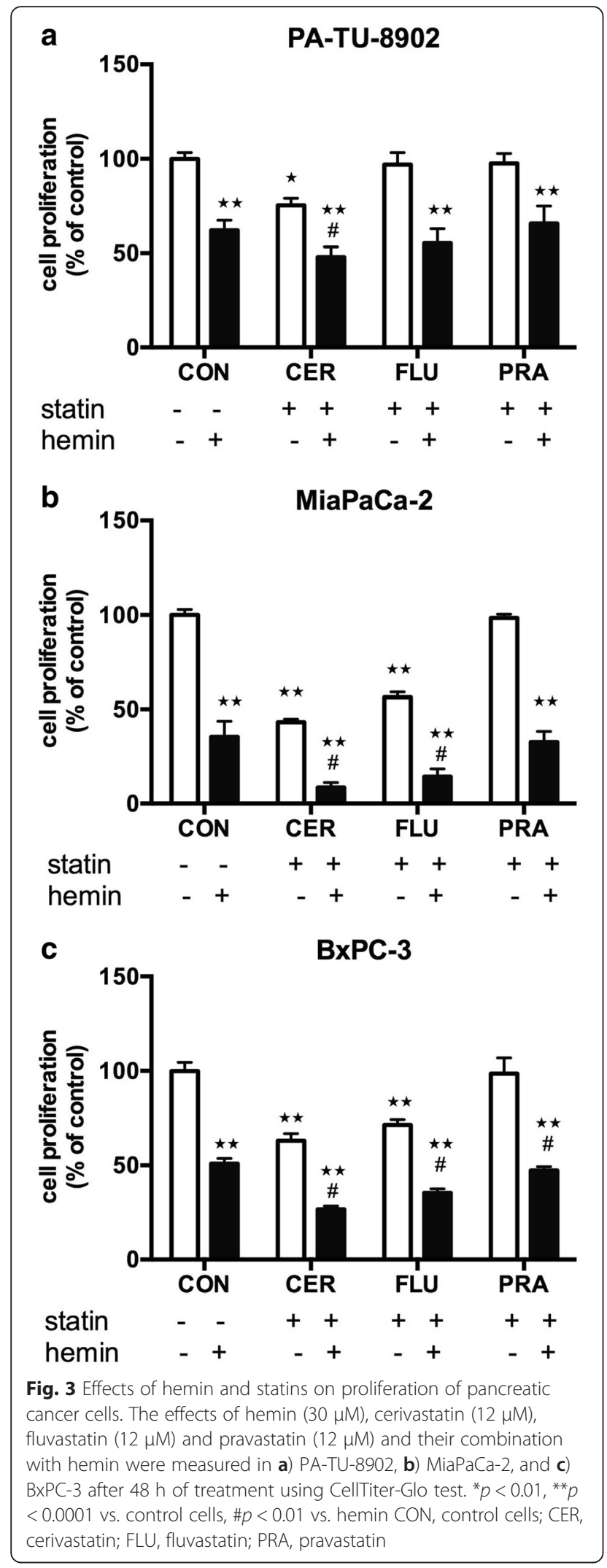

Furthermore, hemin treatment effectively decreased cell proliferation in controls, as well as HMOX-silenced cells, and addition of hemin to cerivastatin endorsed the anti-proliferative effect of cerivastatin (Fig. 5), indicating that these processes are not mediated by HMOX.

\section{Hemin and cerivastatin enhance ROS production and cell death in vitro}

To evaluate the possible mechanism of cell growth suppression, we assessed apoptotic effects of hemin and cerivastatin in PA-TU-8902 and BxPC-3 cells. While there was no notable effect of hemin on early apoptosis, we found a significant increase in the total cell death rate including late apoptotic and post-apoptotic necrotic cells at $48 \mathrm{~h}$ of treatment. In fact, the major increase in cell death was noticed within the first $14 \mathrm{~h}$ of treatment with no significant elevation in the later phase (data not shown). Cerivastatin treatment caused a significant increase in all phases of apoptosis including the early phase (Fig. 6a, b). Since apoptotic pathways may be linked to ROS production, we assessed total ROS production after $48 \mathrm{~h}$ of treatment, and observed a substantial increase in ROS concentration in response to cerivastatin $(314 \pm 53 \%$ and $173 \pm 22 \%$ for PA-TU-8902 and BxPC-3, respectively, $p<0.05$ for both comparisons) and even much higher increase due to hemin $(1394 \pm 372 \%$ and $441 \pm$ $152 \%$ for PA-TU-8902 and BxPC-3, respectively, $p$ $<0.01$ for both comparisons), with a further additive effect of both compounds $(3615 \pm 1043 \%$ and $795 \pm 72 \%$ for PA-TU-8902 and BxPC-3, respectively, $p<0.01$ for both comparisons) (Fig. 6b, c, d). Interestingly, the rate of cell death and ROS production differed between both cell lines suggesting cell death was not in direct relationship with ROS production.

\section{Cerivastatin prevents K-Ras protein trafficking in PA- TU-8902}

Since we previously reported that pravastatin is the only statin not being able to prevent GFP-K-Ras protein from accumulation on the cell membrane in MiaPaCa-2 cells [4], we decided to detect the effect of cerivastatin and pravastatin on localization of GFP$\mathrm{K}$-Ras protein also in the thoroughly tested PA-TU8902 cell line. As demonstrated in Fig. 7, cerivastatin efficiently inhibited GFP-K-Ras protein trafficking from cytoplasm to the cell membrane, while pravastatin was inefficient. Hemin itself, or in combination with statins did not influence GFP-K-Ras trafficking at all (data not shown). 

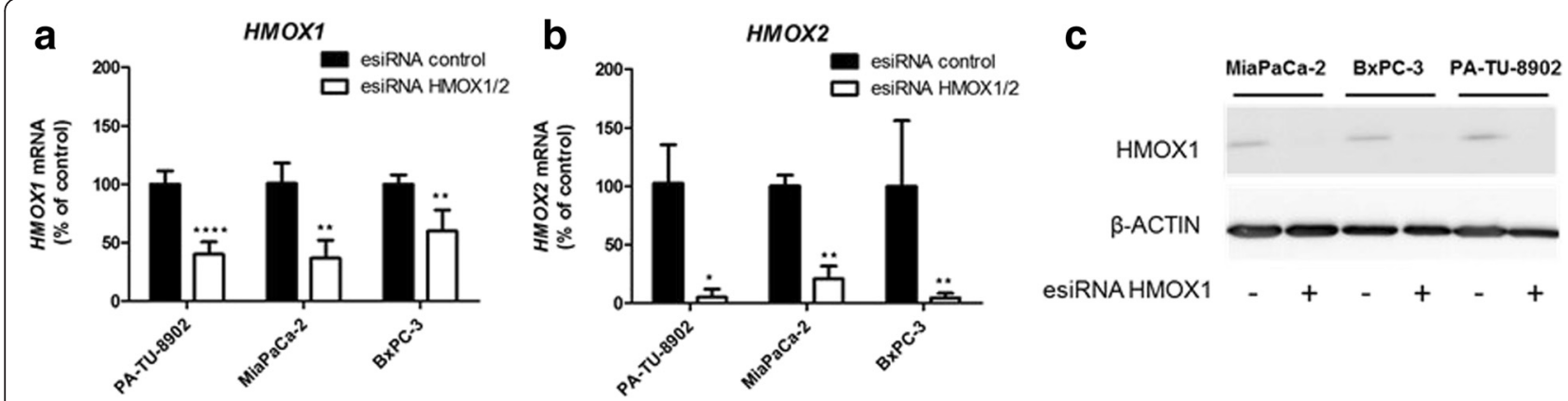

Fig. 4 Effect of esiRNA HMOX1/2 transfection on HMOX expression in pancreatic cancer cells. PA-TU-8902, MiaPaCa-2 and BxPC-3 cells were transfected with esiRNA for HMOX1/HMOX2 or universal control esiRNA and mRNA expression of a) HMOX1 and b) HMOX2 was measured after $24 \mathrm{~h}$. $\mathbf{c}$ HMOX1 protein expression was measured in cells treated with $30 \mu \mathrm{M}$ hemin for $20 \mathrm{~h}$. Hemin treatment was used to upregulate HMOX1 protein expression to cumulate detectable levels of HMOX1 protein. ${ }^{*} p<0.05,{ }^{* *} p<0.01$, ${ }^{* * *} p<0.0001$ vs. esiRNA control cells

\section{Cerivastatin downregulates selected markers of invasiveness}

Among others, poor prognosis of pancreatic cancer is due to early metastases and high invasiveness of this type of tumor. To determine whether cerivastatin treatment affects invasiveness of pancreatic cancer cells, we assessed selected markers previously reported to be associated with metastatic processes in pancreatic cancer, i.e. SPP1 and SOX2. In PA-TU8902 cells, SPP1 mRNA expression was significantly suppressed to $59 \pm 13 \%(p<0.01)$ at $12 \mathrm{~h}$ of treatment with persisting effect of up to $48 \mathrm{~h}(17 \pm 8 \%$ and $40 \pm 20 \%$ at 24 and $48 \mathrm{~h}$, respectively; $p<0.05$; Fig. 8a). Similarly, SOX2 mRNA expression decreased to $25 \pm 9 \%(p<0.01)$ in $12 \mathrm{~h}$ with persisting effect to $48 \mathrm{~h}$ of treatment $(24 \pm 4 \%$ and $61 \pm 13 \%$ at 24 and 48 h; respectively; $p<0.01$, Fig. $8 \mathrm{~b}$ ).

\section{Discussion}

Only little progress has been achieved in the treatment of pancreatic cancer over recent decades [31]. Among multiple tested experimental drugs, statins were shown to display anticancer effects in this malignant disease $[32,33]$. In our study, we investigated a possible relation between statins and the HMOX pathway that play a role in pancreatic carcinogenesis [25].

Even though HMOX upregulation is associated with beneficial effects for cells, its role in carcinogenesis remains controversial [19]. HMOX seems to negatively affect the outcome of treatment [25] and enhance the aggressiveness and progression of pancreatic cancer [34]. Moreover, pancreatic cancer cells were shown to overexpress HMOX1 compared to normal pancreatic tissue $[25,34]$. On the other hand, statins have been previously shown to upregulate $H M O X$ gene expression, and some of their protective effects are believed to be mediated via this pathway [24]. Together with these HMOX1inducing effects, statins simultaneously inhibit pancreatic cancer cell proliferation [4]. In this study, we assessed the overall relationship of particular statins to HMOX regulation in pancreatic cancer cells. We were able to demonstrate no effect of the tested statins on HMOX activity in selected human pancreatic cancer cell lines, despite their remarkable anti-proliferative effects.

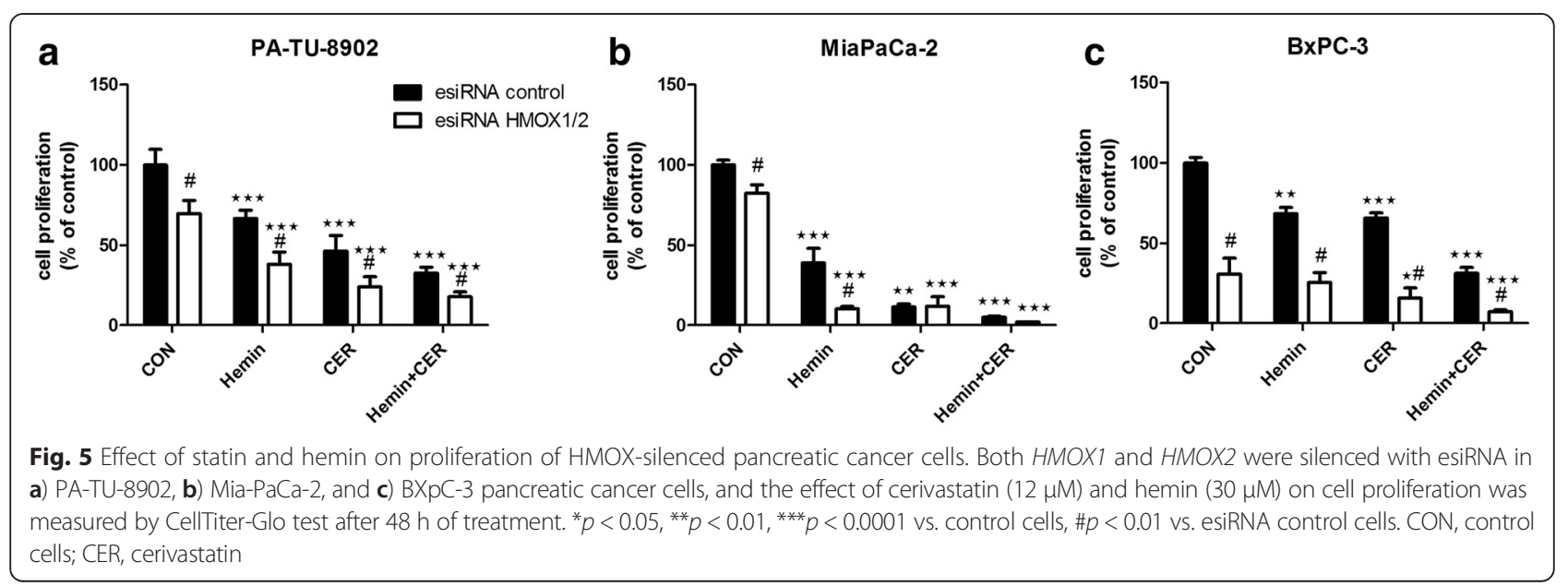



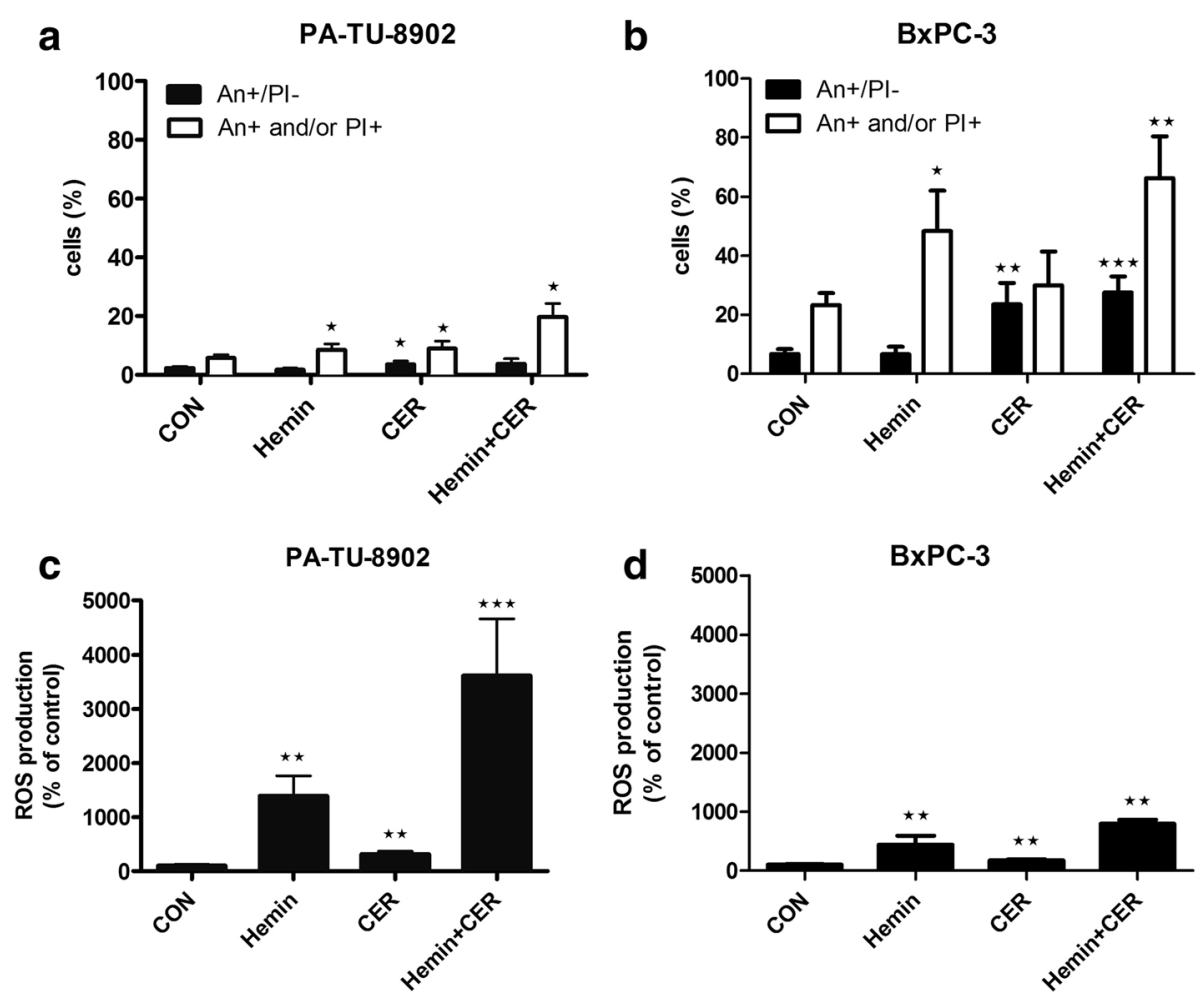

Fig. 6 Effect of hemin and cerivastatin on ROS production and apoptosis in PA-TU-8902 and BxPC-3 pancreatic cancer cells. The effects of hemin $(30 \mu \mathrm{M})$, cerivastatin $(12 \mu \mathrm{M})$ and their combination on apoptosis in a) PA-TU-8902 and b) BxPC-3, and ROS production in c) PA-TU-8902 and d) BxPC-3 were measured after $48 \mathrm{~h}$ of treatment. An+/PI- represents cells in early apoptosis, $\mathrm{An}+$ and/or PI+ represents cells in late apoptosis or already dead. ${ }^{*} p<0.05,{ }^{* *} p<0.01,{ }^{* * *} p<0.0001$ vs. control cells. CON, control cells; CER, cerivastatin

Moreover, suppression of proliferation by cerivastatin treatment persisted also in HMOX1- and HMOX2-silenced cells, indicating that these effects did not depend on the HMOX pathway. Importantly, we noted that $H M O X$ silencing decreased the cell growth, implying an important role of HMOX in pancreatic cancer cell survival. This is in agreement with previous studies $[25,34]$.

To test a possible effect of HMOX on pancreatic carcinogenesis, hemin, a potent HMOX1 inducer [26], was used in further experiments. To our surprise, a significant decrease in cell proliferation was observed in all tested pancreatic cancer cell lines, despite HMOX induction. Moreover, co-treatment of the cells with hemin and statins increased the anti-proliferative effect of the latter. This is most likely due to heminmediated HMOX-independent mechanisms that play a role in cell proliferation and survival. Indeed, we found significant hemin-induced increase in apoptosis, corresponding to considerable increase in ROS production. The same cell growth inhibitory effects were observed even in $H M O X$-silenced cells, further suggesting the independence of hemin bioactivity on the HMOX pathway. Thus, neither statins- nor hemindependent suppression of proliferation involved the
HMOX system; further, induction of HMOX by hemin did not prevent this response of pancreatic cancer cells to the agents. Nevertheless, our data from HMOX1/2 silencing support pro-carcinogenic role of HMOX in pancreatic cancer, consistent with previous clinical observation [25].

Despite the fact that hemin was suggested to contribute to increased colon cancer incidence in red meat eaters [35], other studies shown clear anticancer effects of this compound [36] supporting our findings.

Similarly as in our previous study [4] and as discussed recently [37], we observed remarkable differences in anti-proliferative effects of individual statins, which were dependent also on the cell line used. Both MiaPaCa-2 and PA-TU-8902 cells carry the K-ras mutation in codon 12 [28, 29]. Hamidi and colleagues found that in pancreatic cancer cells, this mutation makes the cells more sensitive to inhibitors of MEK1/ 2 , which is a kinase activated by K-Ras [29]. To extend our study to pancreatic cancer cells lacking the $K$-ras mutation, we also used BxPC-3 cells carrying a wild-type $K$-ras proto-oncogene and overexpressing cyclooxygenase [28]. As expected, MiaPaCa-2 cells were the most susceptible and PA-TU-8902 were the 


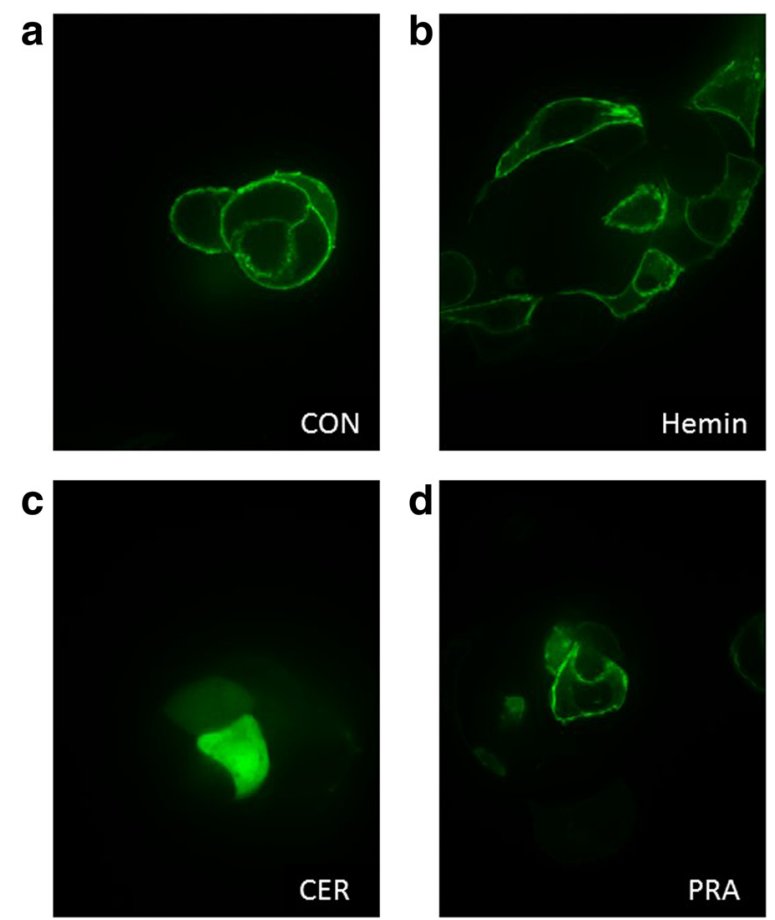

Fig. 7 Effect of hemin and statins on GFP-K-Ras localization in PATU-8902 pancreatic cancer cells. The effect of a) vehicle, $\mathbf{b}$ ) hemin $(30 \mu \mathrm{M}), \mathbf{c})$ cerivastatin $(12 \mu \mathrm{M})$ and $\mathbf{d})$ pravastatin $(12 \mu \mathrm{M})$ on localization of GFP-K-Ras was tested in PA-TU-8902 pancreatic cancer cells transfected with pEGFP-K-RasWT plasmids after $24 \mathrm{~h}$ of treatment. CON, control cells; CER, cerivastatin; PRA, pravastatin most resistant cells to statin treatment in our experiments.

All the statins except pravastatin exerted remarkable growth inhibitory activity in all tested pancreatic cancer cell lines with cerivastatin being the most efficient of these agents. One of the mechanisms possibly involved in anti-carcinogenic effect of cerivastatin might be increased production of ROS, similarly as demonstrated in lymphoma cells [38]. Interestingly, this phenomenon was much more pronounced in hemin-treated cells and, in particular, in cells exposed to hemin together with cerivastatin.

The K-ras pathway is another possible target of statins. In fact, Gbelcova and colleagues demonstrated that statins, except for pravastatin, prevented the GFP-K-Ras protein from its cell membrane localization in MiaPaCa-2 cells [4]. We performed a similar experiment in PA-TU-8902 pancreatic cancer cells, and found that while pravastatin did not affect translocation of the K-Ras protein to the cell membrane, cerivastatin significantly prevented GFP-K-Ras from membrane localization. The K-Ras signaling pathway is essential for metastatic lesion formation and tumor invasiveness [39]. Interestingly, cerivastatin but not pravastatin treatment of PA-TU-8902 cells significantly decreased the expression of SPP1 and $S O X 2$, factors with important role in cancer metastasis and aggressiveness [40, 41]. Meta-analysis of 11 studies revealed that patients with pancreatic cancer have elevated serum levels of SPP1 [42]. Similarly, $S O X 2$ over-expression promotes self-renewal and dedifferentiation of pancreatic cancer cells [43]. In our experiments, both markers were downregulated in pancreatic cancer cells exposed to cerivastatin, pointing to a lowering effect of the statin on the metastatic potential of the cells.

\section{Conclusion}

Our data suggest that anti-proliferative effects of statins are not mediated via HMOX pathway. Cerivastatin, the most efficient statin in our study, was capable of inducing several 'events' involved in carcinogenesis, including apoptosis, ROS production and inhibition of K-Ras trafficking. Hemin treatment not only substantially decreased cell proliferation
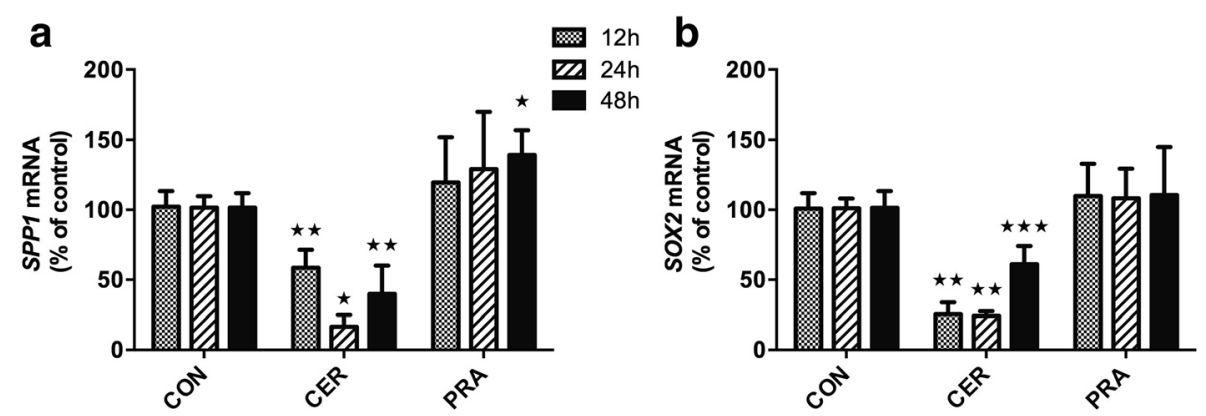

Fig. 8 Effect of cerivastatin and pravastatin on selected markers of invasiveness in PA-TU-8902 pancreatic cancer cells. a) Osteopontin (SPP1) and b) SOX2 mRNA expressions were measured in different time points of treatment with cerivastatin $(12 \mu \mathrm{M})$ and pravastatin $(12 \mu \mathrm{M})$. ${ }^{*} p<0.05$, ${ }^{*} p$ $<0.01,{ }^{* * *} p<0.001$ vs. control cells. CON, control cells; CER, cerivastatin; PRA, pravastatin 
independently on HMOX induction, but enhanced anti-proliferative properties of statins in human pancreatic cancer cells. Our findings support the role of statins as agents with potential anti-pancreatic cancer activities.

\section{Ethics approval and consent to participate Not applicable}

\section{Consent for publication \\ Not applicable}

\section{Availability of data and materials}

The datasets supporting conclusions of this article are included within the article.

\section{Abbreviations}

DMEM: dulbecco's Modified Essential Media; FBS: fetal bovine serum; GFP: green fluorescent protein; HMG CoA: 3-hydroxyl-methylglutaryl coenzyme A; HMOX: heme oxygenase; NADPH: reduced nicotinamide adenine dinucleotide; PGJ2: 15 -deoxy- $\Delta$-12,14-prostaglandin J2;

SPP1: secreted phosphoprotein 1, osteopontin; SOX2: sex-determining region Y-related HMG box 2.

\section{Competing interests}

The authors declare that they have no competing interests.

\section{Authors' contributions}

KV performed the most of cell culture studies, GPCR experiments and drafted the manuscript; SB performed flow cytometry studies; HG performed confocal microscopy studies; LM performed heme oxygenase activity and RNAi experiments; JN was involved in study design, flow cytometry experiments and assisted with drafting of the manuscript: RG contributed to the study design, participated in QPCR experiments, and assisted with drafting of the manuscript; TR contributed to confocal microscopy experiments and assisted with drafting of the manuscript; LV designed the study, supervised all the experiments and assisted with drafting of the manuscript. All authors read and approved the final manuscript.

\section{Acknowledgements}

Not applicable

\section{Funding}

This work was supported by grants by the Czech Ministry of Health (IGA MZ NT14078-3, NT13112-4/2012, RVO-VFN64165/2013), Charles University in Prague (260032-2015, and PRVOUK-P25/LF1/2), as well as by the BIOCEV European Regional Development Fund CZ.1.05/1.100 and VEGA grant 1/ 0407/13 given by the Ministry of Education, Science, Research and Sport of the Slovak Republic

\section{Author details \\ 'Institute of Medical Biochemistry and Laboratory Diagnostics, 1st Faculty of Medicine, Charles University in Prague, Katerinska 32, Prague 2120 00, Czech Republic. ${ }^{2}$ Institute of Biotechnology, Czech Academy of Sciences, Videnska 1083, Prague 4142 20, Czech Republic. ${ }^{3}$ Department of Biochemistry and Microbiology, University of Chemistry and Technology, Technicka 1905/5, Prague 6160 00, Czech Republic. ${ }^{4}$ School of Medical Science, Griffith University, Parklands Avenue, 4222 Southport, QLD, Australia. ${ }^{5}$ Department of Surgery, University Hospital Kralovske Vinohrady and Charles University in Prague, Srobarova 50, Prague 10100 34, Czech Republic. ${ }^{6} 4$ th Department of Internal Medicine, 1st Faculty of Medicine, Charles University in Prague, Katerinska 32, Praque 2120 00, Czech Republic.}

\section{References}

1. Vitek $L$, Lenicek M. Cytoprotective and antiproliferative effects of HMG-CoA reductase inhibitors. Curr Enz Inhib. 2006;2:261-80.

2. Hawk MA, Cesen KT, Siglin JC, Stoner GD, Ruch RJ. Inhibition of lung tumor cell growth in vitro and mouse lung tumor formation by lovastatin. Cancer Lett. 1996;109(1-2):217-22.

3. Agarwal B, Bhendwal S, Halmos B, Moss SF, Ramey WG, Holt PR. Lovastatin augments apoptosis induced by chemotherapeutic agents in colon cancer cells. Clin Cancer Res. 1999;5(8):2223-9.

4. Gbelcova H, Lenicek M, Zelenka J, Knejzlik Z, Dvorakova G, Zadinova M, et al. Differences in antitumor effects of various statins on human pancreatic cancer. Int J Cancer. 2008;122(6):1214-21.

5. Sumi S, Beauchamp RD, Townsend CM, Uchida T, Murakami M, Rajaraman S, et al. Inhibition of pancreatic adenocarcinoma cell growth by lovastatin. Gastroenterology. 1992:103(3):982-9.

6. Kusama T, Mukai M, Iwasaki T, Tatsuta M, Matsumoto $Y$, Akedo $H$, et al. Inhibition of epidermal growth factor-induced RhoA translocation and invasion of human pancreatic cancer cells by 3 hydroxy-3-methylglutaryl-coenzyme a reductase inhibitors. Cancer Res. 2001;61(12):4885-91.

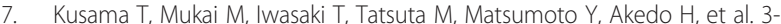
hydroxy-3-methylglutaryl-coenzyme a reductase inhibitors reduce human pancreatic cancer cell invasion and metastasis. Gastroenterology. 2002; 122(2):308-17.

8. Elson CE, Peffley DM, Hentosh P, Mo H. Isoprenoid-mediated inhibition of mevalonate synthesis: potential application to cancer. Proc Soc Exp Biol Med. 1999:221(4):294-311.

9. Sebti SM. Protein farnesylation: implications for normal physiology, malignant transformation, and cancer therapy. Cancer Cell. 2005;7(4):297-300.

10. Pronk GJ, Bos JL. The role of p21ras in receptor tyrosine kinase signalling. Biochim Biophys Acta. 1994;1198(2-3):131-47

11. Hall A. Rho GTPases and the actin cytoskeleton. Science. 1998; 279(5350):509-14.

12. Bondar VM, Sweeney-Gotsch B, Andreeff M, Mills GB, McConkey DJ. Inhibition of the phosphatidylinositol 3'-kinase-AKT pathway induces apoptosis in pancreatic carcinoma cells in vitro and in vivo. Mol Cancer Ther. 2002;1(12):989-97.

13. Almoguera C, Shibata D, Forrester K, Martin J, Arnheim N, Perucho M. Most human carcinomas of the exocrine pancreas contain mutant C-K-ras genes. Cell. 1988:53(4):549-54.

14. Ryter SW, Alam J, Choi AM. Heme oxygenase-1/carbon monoxide: from basic science to therapeutic applications. Physiol Rev. 2006:86(2):583-650.

15. Willis D, Moore AR, Frederick R, Willoughby DA. Heme oxygenase: a novel target for the modulation of the inflammatory response. Nat Med. 1996:2:87-90

16. Lin Q, Weis S, Yang G, Weng YH, Helston R, Rish K, et al. Heme oxygenase-1 protein localizes to the nucleus and activates transcription factors important in oxidative stress. J Biol Chem. 2007;282(28):20621-33.

17. Liu H, Nowak R, Chao W, Bloch KD. Nerve growth factor induces antiapoptotic heme oxygenase-1 in rat pheochromocytoma PC12 cells. J Neurochem. 2003:86(6):1553-63.

18. Wegiel B, Nemeth Z, Correa-Costa M, Bulmer AC, Otterbein LE. Heme oxygenase-1: a metabolic nike. Antioxid Redox Signal. 2014;20(11):1709-22.

19. Was H, Dulak J, Jozkowicz A. Heme oxygenase-1 in tumor biology and therapy. Curr Drug Targets. 2010;11(12):1551-70.

20. Grosser N, Erdmann K, Hemmerle A, Berndt G, Hinkelmann U, Smith G, et al. Rosuvastatin upregulates the antioxidant defense protein heme oxygenase1. Biochem Biophys Res Commun. 2004;325(3):871-6.

21. Grosser N, Hemmerle A, Berndt G, Erdmann K, Hinkelmann U, Schurger S, et al. The antioxidant defense protein heme oxygenase 1 is a novel target for statins in endothelial cells. Free Radic Biol Med. 2004;37(12):2064-71.

22. Lee TS, Chang CC, Zhu Y, Shyy JYJ. Simvastatin induces heme oxygenase-1 - A novel mechanism of vessel protection. Circulation. 2004:110(10):1296-302.

23. Hsu M, Muchova L, Morioka I, Wong RJ, Schroder H, Stevenson DK. Tissuespecific effects of statins on the expression of heme oxygenase- 1 in vivo. Biochem Biophys Res Commun. 2006;343(3):738-44.

24. Muchova L, Wong RJ, Hsu M, Morioka I, Vitek L, Zelenka J, et al. Statin treatment increases formation of carbon monoxide and bilirubin in mice: a novel mechanism of in vivo antioxidant protection. Can J Physio Pharmacol. 2007;85(8):800-10. 
25. Berberat PO, Dambrauskas Z, Gulbinas A, Giese T, Giese N, Kunzli B, et al. Inhibition of heme oxygenase- 1 increases responsiveness of pancreatic cancer cells to anticancer treatment. Clin Cancer Res. 2005;11(10):3790-8.

26. Muchova L, Vanova K, Suk J, Micuda S, Dolezelova E, Fuksa L, et al. Protective effect of heme oxygenase induction in ethinylestradiol-induced cholestasis. J Cell Mol Med. 2015;19(5):924-33.

27. Vreman HJ, Stevenson DK. Heme oxygenase activity as measured by carbon monoxide production. Anal Biochem. 1988;168(1):31-8.

28. Kita K, Saito S, Morioka CY, Watanabe A. Growth inhibition of human pancreatic cancer cell lines by anti-sense oligonucleotides specific to mutated K-ras genes. Int J Cancer. 1999;80(4):553-8.

29. Hamidi H, Lu M, Chau K, Anderson L, Fejzo M, Ginther C, et al. KRAS mutational subtype and copy number predict in vitro response of human pancreatic cancer cell lines to MEK inhibition. Br J Cancer. 2014;111(9):1788-801.

30. Hinkelmann U, Grosser N, Erdmann K, Schroder H, Immenschuh S. Simvastatin-dependent up-regulation of heme oxygenase-1 via mRNA stabilization in human endothelial cells. Eur J Pharm Sci. 2010;41(1):118-24.

31. Vaccaro V, Sperduti I, Vari S, Bria E, Melisi D, Garufi C, et al. Metastatic pancreatic cancer: Is there a light at the end of the tunnel? World J Gastroenterol. 2015;21(16):4788-801.

32. Khurana V, Sheth A, Caldito G, Barkin JS. Statins reduce the risk of pancreatic cancer in humans: a case-control study of half a million veterans. Pancreas. 2007;34(2):260-5.

33. Walker EJ, Ko AH, Holly EA, Bracci PM. Statin use and risk of pancreatic cancer: results from a large, clinic-based case-control study. Cancer. 2015; 121(8):1287-94.

34. Sunamura M, Duda DG, Ghattas MH, Lozonschi L, Motoi F, Yamauchi J, et al. Heme oxygenase-1 accelerates tumor angiogenesis of human pancreatic cancer. Angiogenesis. 2003;6(1):15-24.

35. Glei M, Klenow S, Sauer J, Wegewitz U, Richter K, Pool-Zobel BL. Hemoglobin and hemin induce DNA damage in human colon tumor cells HT29 clone 19A and in primary human colonocytes. Mutat Res. 2006;594(1-2):162-71.

36. Tsuji A, Wang J, Stenzel KH, Novogrodsky A. Immune stimulatory and antitumour properties of haemin. Clin Exp Immunol. 1993:93(3):308-12.

37. Vitek L. Statins and pancreatic cancer: are all statins the same? Am J Gastroenterol. 2009;104(2):525.

38. Qi XF, Zheng L, Lee KJ, Kim DH, Kim CS, Cai DQ, et al. HMG-CoA reductase inhibitors induce apoptosis of lymphoma cells by promoting ROS generation and regulating Akt, Erk and p38 signals via suppression of mevalonate pathway. Cell Death Dis. 2013;4, e518.

39. Collins MA, Pasca di Magliano M. Kras as a key oncogene and therapeutic target in pancreatic cancer. Front Physiol. 2013;4:407.

40. Zhivkova-Galunska M, Adwan H, Eyol E, Kleeff J, Kolb A, Bergmann F, et al. Osteopontin but not osteonectin favors the metastatic growth of pancreatic cancer cell lines. Canc Biol Ther. 2010;10(1):54-64.

41. Sanada Y, Yoshida K, Ohara M, Oeda M, Konishi K, Tsutani Y. Histopathologic evaluation of stepwise progression of pancreatic carcinoma with immunohistochemical analysis of gastric epithelial transcription factor SOX2: comparison of expression patterns between invasive components and cancerous or nonneoplastic intraductal components. Pancreas. 2006;32(2):164-70.

42. Li JJ, Li HY, Gu F. Diagnostic significance of serum osteopontin level for pancreatic cancer: a meta-analysis. Genet Test Mol Biomarkers. 2014; 18(8):580-6.

43. Herreros-Villanueva M, Bujanda L, Billadeau DD, Zhang JS. Embryonic stem cell factors and pancreatic cancer. World J Gastroenterol. 2014;20(9):2247-54.

\section{Submit your next manuscript to BioMed Central and we will help you at every step:}

- We accept pre-submission inquiries

- Our selector tool helps you to find the most relevant journal

- We provide round the clock customer support

- Convenient online submission

- Thorough peer review

- Inclusion in PubMed and all major indexing services

- Maximum visibility for your research

Submit your manuscript at www.biomedcentral.com/submit
Biomed Central 